\title{
Classical Neurotransmitters and Neuropeptides Involved in Major Depression: A Multi-Neurotransmitter System
}

\author{
Werner FM ${ }^{1}$ and Covenas $\mathbf{R}^{2}$ \\ ${ }^{1}$ Euro Akademie Pößneck, Pößneck, Germany and Institute of Neuroscience of Castilla and León (INCYL), Laboratory 14, Salamanca, Spain \\ ${ }^{2}$ Institute of Neuroscience of Castilla and León (INCYL), Laboratory 14, Salamanca, Spain
}

"Corresponding author: Felix-Martin Werner, Euro Akademie Pößneck, Pößneck, Germany and Institute of Neuroscience of Castilla and León (INCYL), Laboratory 14, Salamanca, Spain, Tel: 00493647505520; E-mail: felixm-werner@versanet.de

Rec date: Mar 06, 2014, Acc date: June 06, 2014, Pub date: June 08, 2014

Copyright: (c) 2014 Werner, et al. This is an open-access article distributed under the terms of the Creative Commons Attribution License, which permits unrestricted use, distribution, and reproduction in any medium, provided the original author and source are credited.

\begin{abstract}
In major depression, in both midbrain and hippocampus a hypoactivity of monoamines (serotonin, noradrenalin, dopamine) and a hypoactivity of presynaptic inhibitory neurotransmitters (GABA, glutamate) occurs. Serotonin hypoactivity, which is partly explained by polymorphisms of the serotonin transporter gene, could be as well due to an enhanced presynaptic inhibition carried out by glutaminergic neurons via m5Glu receptors. Noradrenalin hypoactivity is also due to an enhanced presynaptic inhibition exerted, via $\mathrm{GABA}_{\mathrm{B}}$ receptors, by GABAergic neurons. We derived neural networks from direct and reciprocal correlations between neurotransmitters. The neural networks presented here suggest a multimodal antidepressant pharmacotherapy by combining enhancing and antagonistic antidepressant drugs, for example noradrenalin reuptake inhibitors and $\mathrm{GABA}_{B}$ antagonists.
\end{abstract}

Keywords: Antidepressants; Monoamine reuptake inhibitor; Serotonin; Noradrenalin; Dopamine; Neural network

\section{Introduction}

Major depression is a frequent psychiatric disease. Since many patients suffer a form of major depression which cannot be sufficiently treated by antidepressant drugs, the question arises whether new antidepressant drugs, such as $\mathrm{GABA}_{\mathrm{B}}$ antagonists or m5GluR antagonists (antagonists of the subtype 5 of metabotropic glutaminergic receptors) can be combined with current pharmacotherapy, for example SSRI's (selective serotonin reuptake inhibitors) or SNRI's (selective serotonin and noradrenalin reuptake inhibitors). In order to develop this stategy, a neural network involving both the midbrain and hippocampus is here suggested and hence the appropriate combination of new antidepressant drugs with current pharmacotherapy could be justified.

\section{Neural Networks Involved in Major Depression}

Major depression is a psychiatric disease with a prevalence between $5 \%$ and $8 \%$, which is increasing for example as a consequence of stress. It is accompanied by the following symptoms: sadness, lost of energy and interest, an altered circadian rhythm, changes in behavior and mood. When major depression is not treated, it can lead to suicide $[1,2]$. The aim of this short review is to develop neural networks in the brain regions involved in major depression and to establish a functional coherence between classical neurotransmitters and neuropeptides in order to suggest the appropriate combination of antidepressant drugs for achieving a major antidepressant effect [2]. The question arises how the neural networks are developed. The first neural network was developed in the center of the circadian rhythm [3]. From clinical applications, it is known that $5-\mathrm{HT}_{1 \mathrm{~A}}$ agonists and alpha 1 antagonists have a hypnotic effect, while noradrenalin and serotonin exert a postsynaptic excitatory effect [3]. $\mathrm{GABA}_{\mathrm{A}}$ agonists and NMDA antagonists have a sedating, hypnotic and partly anaesthetic effect, while GABA and glutamate have a presynaptic inhibitory effect. It is known that $\mathrm{GABA}_{\mathrm{A}}$ agonists inhibit presynaptically alpha1 noradrenergic neurons, and that NMDA antagonists inhibit serotonergic neurons. From these findings, most of them taken from laboratarory animals, a hypothetic neural network involving the centre of the circadian rhythm were developed: serotonin and noradrenalin have alternating concentrations during the day; serotonin is preponderant during the sleep and noradrenalin during the day [3]. The neural network has been confirmed by animal experimentation and clinical application, however an experimental histological proof has not yet been done.

Experimentally, neural networks were examined in depressant patients using fMRI techniques. In these studies, antidepressant pharmacotherapy increased responses to positive stimuli the in the nucleus accumbens, the putamen, the medial prefrontal cortex and the midbrain [4]. In major depression, neural networks are being examined through fMRI techniques. With this technique, the reward neural networks including the insula, the precuneus, the cuneus, the prefrontal cortex and the inferior parietal lobule play a role in improving depressant symptoms [5] (Figure 1).

\section{Antidepressant Pharmacotherapy: How to Improve the Therapeutic Effect?}

In major depression a hypoactivity of the monoamines exerting a postsynaptic excitatory action and a hyperactivity of presynaptic inhibitory neurotransmitters occurs. In the midbrain, serotonin exerts its function via $5-\mathrm{HT}_{1 \mathrm{~A}}$ receptors and noradrenalin via alpha1 receptors, whereas in the hippocampus dopamine exerts its action via $\mathrm{D}_{2}$ receptors. In the midbrain, the presynaptic inhibitory neurotransmitters GABA and glutamate act respectively via $G_{A B A}$ and the subtype 5 of the metabotropic glutaminergic receptors (m5GluR) [6,7]. Hypoactivity of monoamines is due to alterations of 
the corresponding transporter genes. Acetylcholine is as well involved via nicotinic and muscarinergic cholinergic receptors and antagonizes the action of serotonin [2]. Moreover, in major depression, alterations of the following peptides have been described: corticotropin releasing factor (CRH) (increased in the hypothalamus), thyrotropin releasing factor (decreased in serum levels), substance P (increased levels in the hippocampus), galanin (decreased in the hypothalamus), opioids (decreased in thalamus and cortex) and orexins (decreased levels in the cerebrospinal fluid) [1].

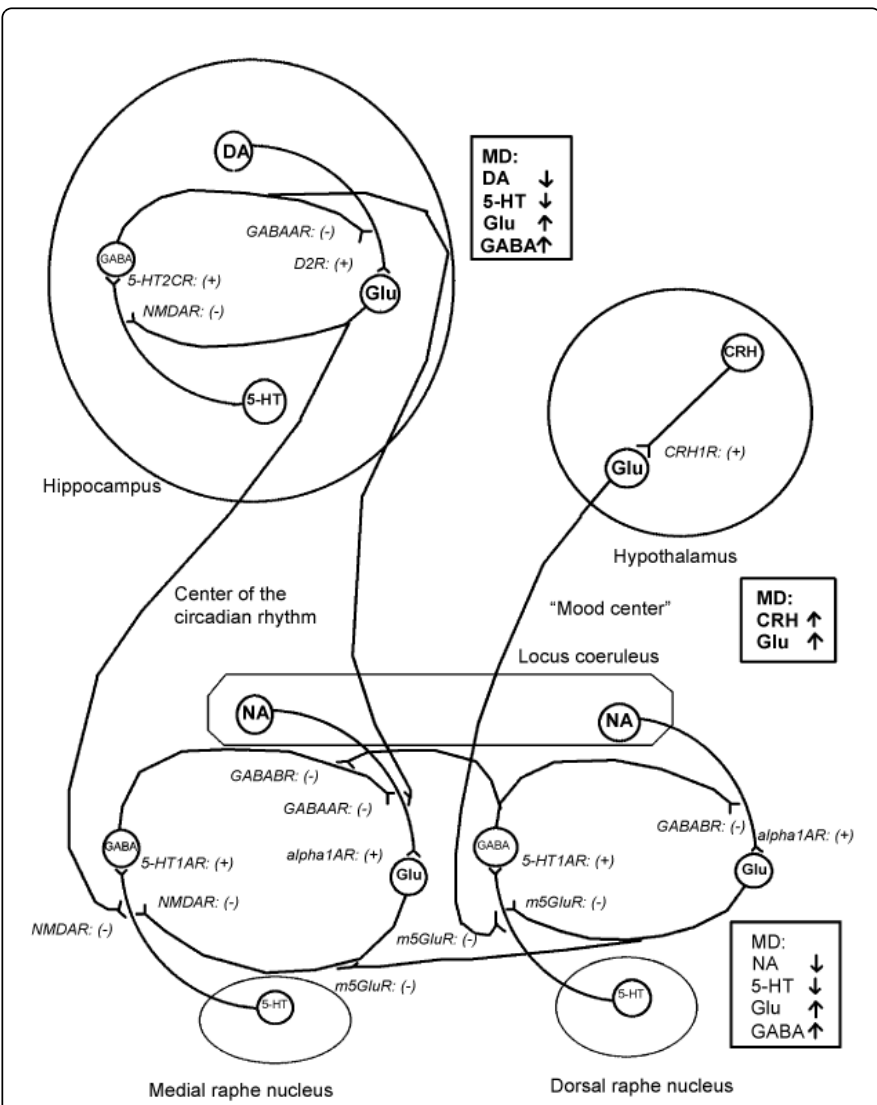

Figure 1: Neural networks, classical neurotransmitters and neuropeptides involved in major depression. Alpha1AR: alpha-1A receptor, a subreceptor of the noradrenergic receptor; $\mathrm{CRH}$ : corticotropin-releasing hormone; CRH1R: CRH-1 receptor, a subreceptor of the $\mathrm{CRH}$ receptor; DA: dopamine; D2R: D2 receptor: a subreceptor of the dopaminergic receptor; GABA: gamma-aminobutyric acid; GABAAR: GABAA receptor: a subreceptor of the GABAergic receptor; GABABR: GABAB receptor: a subreceptor of the GABAergic receptor; Glu: glutamate; 5-HT: serotonin; 5-HT1AR: 5-HT1A receptor: a subreceptor of the serotonergic receptor; 5-HT2CR: 5-HT2C receptor: a subreceptor of the serotonergic receptor; m5GluR: m5Glu receptor: a subreceptor of the metabotropic glutaminergic receptor; $\mathrm{MD}$ : major depression; NA: noradrenalin; NMDAR: N-methyl-Daspartate receptor. A plus mark indicates a postsynaptic excitatory impulse, and a minus mark indicates a presynaptic inhibitory impulse. The arrow upwards denotes increase, and the arrow downwards denotes decrease
Thus, we develop neural networks in the brain regions involved in major depression. These neural networks can be described as follows: in the hippocampus, dopaminergic neurons transmit a weak activating impulse via $D_{2}$ receptors to glutaminergic neurons which strongly inhibit serotonergic neurons through NMDA (N-methyl-D-aspartate) receptors [6]. Serotonergic neurons transmit a weak postsynaptic impulse to $\mathrm{GABAergic}$ neurons via $5-\mathrm{HT}_{2 \mathrm{C}}$ receptors which strongly inhibit dopaminergic neurons via $\mathrm{GABA}_{\mathrm{A}}$ receptors. Glutaminergic and GABAergic neurons send projections to the corresponding neurons of the centre of the circadian rhythm located in the midbrain. In the "mood centre", noradrenergic neurons transmit a weak activating impulse via alpha 1 receptors to glutaminergic neurons which strongly inhibit serotonergic neurons via the m5GluR. The serotonergic neurons transmit a weak postsynaptic impulse via 5$\mathrm{HT}_{1 \mathrm{~A}}$ receptors to GABAergic neurons which strongly inhibit noradrenergic neurons through $\mathrm{GABA}_{\mathrm{B}}$ receptors [7]. In the hypothalamus, $\mathrm{CRH}$ containing neurons which have a high activity transmit a strong activating impulse through $\mathrm{CRH}_{1}$ receptors to glutaminergic neurons which inhibit serotonergic neurons in the dorsal raphe nucleus via $\mathrm{m} 5 \mathrm{Glu}$ receptors and reduce serotonin levels [2].

The current treatment of major depression is the administration of monoamine reuptake inhibitors. Selective serotonin reuptake inhibitors have a restricted effect. The noradrenalin and serotonin reuptake inhibitor, venlafaxine, exerts an improved antidepressant effect. Progress in the antidepressant effect has been achieved by the noradrenalin reuptake inhibitor ruboxetine and the selective dopamine and noradrenalin reuptake inhibitor bupropion, which exert a potent antidepressant effect and treat the decreased positive effects [1].

It should be examined whether additional antidepressant pharmacotherapies could be applied, for example:

- $\mathrm{GABA}_{\mathrm{B}}$ antagonists or m5GluR antagonists, which enhance noradrenaline or serotonin levels,

- NMDA antagonists, which enhance serotonin levels,

- $5-\mathrm{HT}_{1 \mathrm{~A}}$ or $5-\mathrm{HT}_{2 \mathrm{C}}$ agonists,

- Alpha1 or $\mathrm{D}_{2}$ agonists,

CRH1 receptor antagonists, which enhance serotonin levels through a reduced glutaminergic presynaptic inhibition via m5Glu receptors, have been shown to have a low clinical effect $[1,2]$.

\section{Are Neural Networks Useful for Improving an Antidepressant Pharmacotherapy?}

It is important to examine neural networks in the brain regions involved in major depression in order to optimize a multimodal antidepressant pharmacotherapy. The clinical effects of proving such neural networks could be the following: If it was proved that m5GluR antagonists and NMDA antagonists enhance serotonergic neurotransmission through a reduced presynaptic inhibition [4]. Then it could be examined in clinical trials whether these antidepressant drugs, for example MTEP (3-[methyl-1,3-thiazol-4-yl)ethynyl]pyridine), which could be combined with selective serotonin reuptake inhibitors, might achieve a major antidepressant effect. Besides, it can be investigated whether $\mathrm{GABA}_{\mathrm{B}}$ antagonists, which enhance noradrenalin levels $[7,8]$, could be combined with antidepressant drugs which inhibit noradrenalin reuptake. In sum, some patients 
Citation: Werner FM, Covenas R (2014) Classical Neurotransmitters and Neuropeptides Involved in Major Depression: A Multi-Neurotransmitter System. J Cytol Histol 5: 253. doi:10.4172/2157-7099.1000253

Page 3 of 3

suffering from severe major depression might profit from a multimodal pharmacotherapy.

\section{Conclusion}

Major depression is the most frequent psychiatric disease, and treatment-resistant forms of major depression occur more often, which cannot be treated with current antidepressant drugs. Derived from hypnotic and sedating drugs acting in the centre of the circadian rhythm and animal experimentation, we developed neural networks including four neurotransmitters in the midbrain and the hippocampus. Monoamine hypoactivity is due to alterations of the monoamine transporter genes, but also to an increased presynaptic inhibition through GABAergic and glutaminergic neurons. The developed neural networks, the histological proof of which still has to be done, enables to combine current antidepressant drugs with recently developed drugs, for example, selective serotonin reuptake inhibitors with NMDA antagonists or m5GluR antagonists.

\section{References}

1. Werner FM, Coveñas R (2010) Classical neurotransmitters and neuropeptides involved in major depression: a review. Int J Neurosci 120: 455-470.
2. Werner FM, Coveñas R (2013) Classical neurotransmitters and neuropeptides involved in major depression in a multi-neurotransmitter system: a focus on antidepressant drugs. Curr Med Chem 20: 4853-4858.

3. Werner FM, Coveñas R (2011) Classical neurotransmitters involved in the regulation of the sleep-wake cycle and thereof derived pharmacological options. Deutsche Gesellschaft für Neurologie, P477.

4. Ma Y (2014) Neuropsychological mechanism underlying antidepressant effect: a systematic meta-analysis. Mol Psychiatry.

5. Zhang WN, Chang SH, Guo LY, Zhang KL, Wang J (2013) The neural correlates of reward-related processing in major depressive disorder: a meta-analysis of functional magnetic resonance imaging studies. J Affect Disord 151: 531-539.

6. Zhang WN, Chang SH, Guo LY, Zhang KL, Wang J (2013) The neural correlates of reward-related processing in major depressive disorder: a meta-analysis of functional magnetic resonance imaging studies. J Affect Disord 151: 531-539.

7. Pomierny-Chamiolo, Poleszak E, Pilc A, Nowak G (2010) NMDA but not AMPA glutamatergic receptors are involved in the antidepressant-like activity of MTEP during the forced swim test in mice. Pharmacol Rep 62: 1186-1190.

8. Nowak G, Partyka A, Pałucha A, Szewczyk B, Wierońska JM, et al. (2006) Antidepressant-like activity of CGP 36742 and CGP 51176, selective GABAB receptor antagonists, in rodents. Br J Pharmacol 149: 581-590. 